\title{
Nefropatia Diabética e Doença Cardíaca
}

revisão

\author{
JoRGE LUIz GROSS \\ SANDRa PINHO SILVEIRO \\ LUIS HeNRIQUe CANANI \\ ROGÉRIO FRIEDMAN \\ CRISTIANE BAUERMANN LEITÃo \\ Mirela Jobim de AzeVEdo
}

Serviço de Endocrinologia do
Hospital de Clínicas de
Porto Alegre, RS.

Recebido em 10/01/07

Aceito em 15/01/07
RESUMO

Pacientes em diferentes estágios de nefropatia diabética (ND) apresentam freqüentemente comprometimento cardíaco expresso por isquemia miocárdica e/ou cardiomiopatia diabética. Estas alterações já estão presentes em estágios iniciais da ND e provavelmente mesmo antes de a excreção urinária de albumina (EUA) atingir níveis tradicionalmente diagnósticos de microalbuminúria. As alterações cardíacas são responsáveis por uma proporção significativa de mortes nos pacientes com ND e podem ser reduzidas através de intervenção nos múltiplos fatores de risco cardiovascular encontrados nesses pacientes. A avaliação de doença cardíaca deve idealmente ser realizada em todos os pacientes com qualquer grau de ND através de métodos específicos para detectar isquemia e disfunção miocárdica, além do emprego rotineiro da monitorização ambulatorial da pressão arterial em $24 \mathrm{~h}$. Em pacientes com aterosclerose avançada também devem ser avaliadas outras artérias (carótidas, aorta, renais). O tratamento rigoroso da hipertensão arterial, o uso de fármacos cardioprotetores, o tratamento da dislipidemia e da anemia, assim como o emprego de medicamentos anti-plaquetários, poderão reduzir a elevada mortalidade cardiovascular na ND. (Arq Bras Endocrinol Metab 2007; 51/2:244-256)

Descritores: Nefropatia diabética; Doença cardiovascular; Cardiopatia isquêmica; Fatores de risco; Tratamento

\begin{abstract}
Diabetic Nephropathy and Cardiac Disease.

Patients in different stages of diabetic nephropathy (DN) frequently present cardiac disease expressed by myocardial ischemia and/or diabetic cardiomyopathy. These changes are already present at early stages of DN, probably even before urinary albumin excretion (UAE) reaches the traditionally diagnostic levels of microalbuminuria. The cardiac changes are responsible for a significant proportion of the increased death rates in patients with DN and can be reduced through multiple intervention on the several risk factors present in these patients. Cardiac disease assessment should ideally be performed in every patient, irrespective of renal status, through specific methods to detect ischemia and myocardial dysfunction, besides routinely performing 24-h ambulatory blood pressure monitoring. In patients with advanced atherosclerosis, other arteries (aorta, carotid, renal) should be evaluated as well. Intensive treatment of arterial hypertension, and use of cardioprotective drugs, correction of the associated dyslipidemia and anemia, and use of antiplatelet agents can reduce the elevated cardiovascular mortality in patients with DN. (Arq Bras Endocrinol Metab 2007;51/2:244-256)
\end{abstract}

Keywords: Diabetic nephropathy; Cardiovascular disease; Ischemic heart disease; Risk factors; Treatment 
A ASSOCIAÇÃO ENTRE NEFROPATIA diabética (ND) e doença cardíaca é bem conhecida já há muitas décadas. No início dos anos 80, ficou bem definido que os pacientes diabéticos tipo 1 ou tipo $2 \mathrm{com}$ proteinúria apresentavam um aumento de 3 a 4 vezes da mortalidade, principalmente por causas cardíacas $(1,2)$. Mais recentemente, o United Kingdom Prospective Diabetes Study (UKPDS) mostrou que há um aumento progressivo da mortalidade de pacientes diabéticos à medida que aumenta o grau de comprometimento renal, podendo chegar a uma taxa de mortalidade de $19 \%$ ao ano nos pacientes com insuficiência renal (3). Além disto, a presença de ND está associada a um aumento da proporção de pacientes com cardiopatia isquêmica assintomática (4).

A ND tem sido didaticamente caracterizada em estágios de acordo com os valores de excreção urinária de albumina (EUA) em microalbuminúria e macroalbuminúria. Os valores de ponto de corte em diferentes tipos de coleta para caracterizar esses estágios estão descritos na tabela 1 (2). Atualmente, alguns autores admitem que essa classificação possa ser arbitrária, já que evidências vêm se acumulando de que a EUA representa um risco contínuo para hipertensão arterial (5), desenvolvimento de macroalbuminúria (6) e mortalidade cardiovascular (7).

A doença cardíaca em pacientes diabéticos é usualmente atribuída à isquemia miocárdica decorrente de um processo aterosclerótico coronário acelerado e mais extenso. Pacientes diabéticos são mais predispostos a desenvolverem insuficiência cardíaca congestiva, independentemente da presença de hipertensão arterial ou doença coronariana (8). Há numerosas evidências clínicas que sugerem a existência de anormalidades específicas da estrutura e função do miocárdio em pacientes diabéticos denominada de cardiomiopatia diabética (9).

O objetivo do presente trabalho é revisar os principais aspectos relacionados à doença cardíaca em pacientes diabéticos, levando-se em consideração os diferentes estágios da ND.

\section{MICROALBUMINÚRIA}

A presença de microalbuminúria está fortemente ligada à doença cardiovascular (DCV) $(10,11)$, entretanto a natureza desta relação não é completamente estabelecida. O aumento da EUA pode ser um marcador de dano endotelial (12) ou representar dano glomerular de ND em fase inicial.

\section{Excreção urinária de albumina como um indicador de risco}

Indivíduos com DM tipo 2 e microalbuminúria têm um risco 2,4 vezes maior de morte cardiovascular do que indivíduos sem microalbuminúria (13). Há um aumento progressivo do risco com o aumento da EUA, mesmo nos indivíduos classificados como normoalbuminúricos $(14,15)$, sugerindo que o risco conferido pelo aumento da EUA seja contínuo.

\section{Fator de risco comum à microalbuminúria e doença cardiovascular}

Pacientes microalbuminúricos, à semelhança dos pacientes com macroalbuminúria, apresentam uma agregação de fatores de risco: ausência de descenso noturno da pressão arterial, aumento da pressão arterial (16), dos triglicerídeos, do colesterol total e do LDL e IDL colesterol (17), aumento da proporção sérica de ácidos graxos saturados (18) e da presença dos componentes da síndrome metabólica (19) e disfunção endotelial (20). Mais freqüentemente, esses pacientes apresentam retinopatia diabética, amputação de membros inferiores, doença arterial coronariana e acidente vascular encefálico. A função renal medida pela taxa de filtração glomerular (TFG) usualmente se mantém estável nos pacientes microalbuminúricos, ainda que possa apresentar um declínio significativo em alguns pacientes (21).

Tabela 1. Valores de albuminúria utilizados para o diagnóstico de nefropatia diabética.

\begin{tabular}{lcccc}
\hline Estágio & $\begin{array}{c}\text { Urina com } \\
\text { tempo } \\
\text { marcado } \\
(\boldsymbol{\mu} \mathbf{g} / \mathbf{m i n})\end{array}$ & $\begin{array}{c}\text { Urina de 24 h } \\
(\mathbf{m g} / \mathbf{2 4} \mathbf{~ h})\end{array}$ & $\begin{array}{c}\text { Amostra isolada de urina } \\
\text { concentração } \\
(\mathbf{m g} / \mathbf{l})\end{array}$ & $\begin{array}{c}\text { índice alb-cr } \\
\text { (mg/g) }\end{array}$ \\
Normoalbuminúria & $<20$ & $<30$ & $<17$ & $<30$ \\
Microalbuminúria & 20 a 199 & 30 a 299 & 17 a 173 & 30 a 299 \\
Macroalbuminúria & $\geq 200$ & $\geq 300$ & $\geq 174$ & $\geq 300$ \\
& & $(\geq 500 *)$ & $(\geq 430 *)$ & \\
\hline
\end{tabular}

* valores de proteína total 


\section{EUA como alvo terapêutico}

Considerando que um aumento da EUA representa um risco cardiovascular $(\mathrm{CV})$ contínuo, é lógico supor que a redução dos seus níveis se traduziria em redução de eventos CV. No entanto, esta é ainda uma questão em aberto. No estudo LIFE, a diminuição da EUA em indivíduos hipertensos com hipertrofia de ventrículo esquerdo e sem DM esteve associada à menor taxa de eventos CV (IAM, AVC e morte), independentemente do efeito nos níveis pressóricos (22). No estudo Prevention of Renal and Vascular End-Stage Disease (PREVEND) observou-se diminuição significativa da EUA e uma tendência de diminuição de mortalidade por DCV em indivíduos microalbuminúricos (23). A crítica a essas evidências é que estes estudos não foram desenhados para avaliar o efeito isolado na EUA, e não se pode descartar que o efeito protetor seja secundário ao fármaco utilizado ou a outros fatores não avaliados. Esta resposta talvez esteja disponível em 2008 com os dados do estudo prospectivo Avoiding Cardiovascular Events through Combination Therapy in Patients with Systolic Hypertension (ACCOMPLISH) (24), que tem como um dos objetivos a avaliação do efeito da diminuição da EUA nos desfechos CVs.

\section{MACROALBUMINÚRIA}

Nesta fase da doença renal, a mortalidade por DCV é maior do que na fase de microalbuminúria, podendo chegar a 4,6\% ao ano (3). De uma maneira geral, há um aumento dos fatores de risco descritos na etapa anterior: os pacientes são mais freqüentemente homens, a maioria tem hipertensão arterial e são portadores de retinopatia diabética e, freqüentemente, de neuropatia autonômica (4). As anormalidades lipídicas são muito comuns e os valores de triglicerídeos podem ser extremamente aumentados quando a proteinúria atinge níveis nefróticos. Há uma perda progressiva da função renal que aumenta o risco de morte CV (25). Nos pacientes com DM tipo 1 proteinúricos sem tratamento adequado, ocorre uma redução nos valores de TFG da ordem de $1,2 \mathrm{ml} / \mathrm{min} / \mathrm{mês}$ (26). A perda de função renal em pacientes com DM tipo 2 é mais heterogênea, podendo ocasionalmente ser mais lenta, com valor de declínio da TFG de aproximadamente $0,5 \mathrm{ml} / \mathrm{min} / \mathrm{mês}$, embora em alguns pacientes a função renal possa se manter estável por longo tempo $(27,28)$. A soma destes fatores de risco se traduz por um aumento do número de estenoses nas artérias coronárias e da placa aterosclerótica conforme demonstrado recentemente em um estudo realizado com angiorressonância magnética em pacientes com DM tipo l e proteinúria (29).

\section{INSUFICIÊNCIA RENAL}

Durante a fase de insuficiência renal, há um aumento acentuado da mortalidade cardiovascular em pacientes diabéticos. Neste estágio, os pacientes se tornam particularmente vulneráveis a eventos $\mathrm{CV}$, pois a diminuição da função renal por si só já é um indicador de aumento de mortalidade que se adiciona aos fatores de risco próprios do diabetes. Além disso, a progressão de outras complicações crônicas, especialmente a neuropatia autonômica, favorece o desenvolvimento de eventos cardiovasculares fatais ou não-fatais $(30)$.

Quando os pacientes iniciam a substituição da função renal, usualmente por hemodiálise, a mortalidade se torna muita acentuada, de tal forma que aproximadamente $50 \%$ dos pacientes morrem em 2 anos (31). O principal fator determinante da mortalidade nesses pacientes é a presença de disfunção diastólica do tipo pseudonormal ou restritiva independentemente do controle metabólico e da presença de isquemia miocárdica (32). A menor complacência do ventrículo esquerdo provavelmente diminui a adaptação desses pacientes às bruscas mudanças de volume que comumente ocorrem entre as sessões de diálise.

\section{AVALIAÇÃO DA DOENÇA CARDÍACA EM PACIENTES COM ND}

Pacientes em qualquer estágio da ND devem realizar uma avaliação cardíaca que contemple tanto o diagnóstico de isquemia miocárdica como o da função cardíaca.

\section{Avaliação da doença coronariana em pacientes com ND}

A avaliação da presença de isquemia miocárdica é usualmente realizada através de procedimentos bem estabelecidos como a história de angina típica ou atípica, infarto agudo do miocárdio no passado, ECG de repouso, ECG de esforço, cintilografia miocárdica e ecocardiograma de estresse. Quando positivos, estes testes indicam a presença de doença aterosclerótica coronariana com obstrução de pelo menos $75 \%$ da luz dos vasos.

Outros métodos não-invasivos podem estabelecer a presença de aterosclerose avançada. Entre estes está a pesquisa de cálcio coronário por tomografia computadorizada (33). A ausência de cálcio coronário torna pouco provável a presença de aterosclerose coronariana, enquanto que aumento da quantidade de cálcio se correlaciona com a gravidade do processo aterosclerótico. Em pacientes com DM 
tipo 2 sem sintomas de isquemia miocárdica, a medida de cálcio coronário tem-se mostrado superior à previsão de eventos cardiovasculares pelos escores de Framingham ou do UKPDS. A principal utilidade clínica deste método é melhor estratificar os pacientes quanto ao risco de eventos $\mathrm{CV}$ e definir a avaliação cardiovascular que deve ser realizada. Pacientes com aumento dos níveis de cálcio coronariano devem realizar uma avaliação da presença de isquemia miocárdica e, mesmo que esta seja negativa, recomenda-se intensificar o controle dos fatores de risco existentes (33).

\section{Avaliação do comprometimento aterosclerótico em outros locais e estenose da artéria renal}

$\mathrm{Na}$ presença de aterosclerose coronariana importante, sugere-se que também sejam avaliados outros locais freqüentes de doença macrovascular como as artérias carótidas, a aorta abdominal e as artérias dos membros inferiores, utilizando métodos específicos e apropriados. Um aspecto importante que deve ser lembrado é a possibilidade de haver estenose das artérias renais. Estenose crítica da artéria renal (> 70\%) ocorre em aproximadamente $17 \%$ dos pacientes DM tipo 2 hipertensos (34) e pode estar associada à HAS e insuficiência renal (nefropatia isquêmica). Nesses pacientes, o uso de inibidores da enzima conversora da angiotensina (IECA) ou antagonistas do receptor da angiotensina II (ARA II) pode reduzir a pressão de filtração transcapilar, levando à insuficiência renal aguda ou crônica, especialmente se a estenose da artéria renal for bilateral ou ocorrer na presença de rim único. Um aumento de creatinina sérica superior a $50 \%$ após o uso desses agentes sugere a presença de estenose de artéria renal (35). Outros dados sugestivos são redução da função renal com proteinúria mínima ou ausente, ausência de retinopatia ou presença de retinopatia diabética mínima, presença de doença macrovascular em outros sítios (coronárias, carótidas, artérias periféricas), presença de sopros vasculares (especialmente femoral) e rins com redução de tamanho e assimétricos à ultra-sonografia (35).

A obstrução de artéria renal, ainda que não crítica, está associada com um mau prognóstico renal e cardiovascular. Assim sendo, por ocasião de estudos invasivos da circulação coronariana, a realização simultânea de cateterismo das artérias renais deve ser cogitada quando: 1) o paciente apresentar hipertensão de início precoce (<30 anos) ou hipertensão grave após os 55 anos; 2) a hipertensão for acelerada, resistente ou maligna; 3 ) há atrofia ou assimetria renal $(>1,5 \mathrm{~cm}$ de diferença entre rins) sem outra causa; 4) houver edema pulmonar súbito ou sem causa aparente; 5) houver disfunção renal inexplicada, incluindo início de terapia de substituição renal; 6) houver piora de função renal ou desenvolvimento de azotemia com o início de um IECA ou ARA II; 7) a doença coronariana for de múltiplos vasos ou houver macroangiopatia periférica múltipla; 8) houver insuficiência cardíaca congestiva ou angina refratária inexplicada (36).

Ressonância magnética com angiografia é o método de escolha para rastrear estenose de artéria renal em pacientes com DM. Outras opções, mas com menor sensibilidade como método diagnóstico, são a cintilografia renal com administração de captopril e a ultra-sonografia das artérias renais com Doppler. A cintilografia renal com captopril apresenta limitações nos pacientes com função renal diminuída (creatinina sérica $>2 \mathrm{mg} / \mathrm{dl}$ ) e a ultra-sonografia com Doppler é fortemente dependente da experiência do radiologista (37). A revascularização raramente cura a hipertensão, mas melhora ou estabiliza a função renal em pacientes com insuficiência renal crônica (37).

\section{Nefropatia após contraste}

Muitos pacientes diabéticos necessitarão realizar exames com contraste iodado devido à prevalência elevada de doença macrovascular, sendo expostos ao risco de apresentarem a nefropatia induzida pelo contraste. Esta entidade é definida como a ocorrência de um aumento da creatinina sérica de $0,5 \mathrm{mg} / \mathrm{dl}$ ou um aumento de $25 \mathrm{a}$ $50 \%$ em relação a seus níveis basais após a administração endovenosa ou intra-arterial de contraste iodado (38). O principal fator determinante é a presença de doença renal prévia. Pode ocorrer em cerca de $8 \%$ dos pacientes com função renal normal. Outros fatores de risco são uso de contraste iônico, idade maior do que 75 anos, diminuição da volemia, presença de insuficiência cardíaca, cirrose, proteinúria e uso concomitante de diuréticos e agentes antiinflamatórios. Essa diminuição da função renal atinge o pico em 3 dias, é usualmente transitória e os valores de creatinina voltam ao normal em 10 dias. As medidas de prevenção incluem escolha de agentes de contraste menos nefrotóxicos (não-iônicos) e utilização de doses menores; otimização da hidratação do paciente, uso de drogas antioxidantes e suspensão temporária de drogas com potencial nefrotóxico (39). O melhor regime de hidratação é a infusão de cloreto de sódio a $0,9 \% 1 \mathrm{ml} / \mathrm{kg} / \mathrm{h}$ por 24 horas, iniciado $12 \mathrm{~h}$ antes do procedimento. Em pacientes ambulatoriais, a hidratação oral pode ser realizada, devendo-se orientar a ingestão mínima de 2 litros de água ou soro fisiológico, 12 horas antes do procedimento (38). O uso de acetilcísteína nas doses de 600 $\mathrm{mg} \mathrm{12/12} \mathrm{h} \mathrm{via} \mathrm{oral,} \mathrm{com} \mathrm{início} \mathrm{de} \mathrm{uso} 12 \mathrm{~h}$ antes e no dia do procedimento, é recomendado por alguns autores, embora o benefício seja duvidoso (40). 


\section{Ecocardiograma}

A realização do ecocardiograma, além de ser uma alternativa para a avaliação de cardiopatia isquêmica em pacientes incapazes de realizar exercício quando realizado sob estresse farmacológico, é extremamente útil em condições de repouso para os pacientes com ND. Permite detectar áreas com menor mobilidade da parede dos ventrículos que representam provavelmente locais prévios de infarto e/ou fibrose. Além disto, é possível estabelecer a presença de disfunção diastólica do ventrículo esquerdo através de estudo com Doppler, a qual pode surgir precocemente e antes da disfunção sistólica (41). A disfunção diastólica é usualmente categorizada nos seguintes estágios: 1) déficit de relaxamento, 2) padrão pseudonormal e 3) padrão restritivo. As duas últimas categorias, especialmente o padrão restritivo, estão associadas a aumento de mortalidade. Essas alterações funcionais estão associadas a níveis de glico-hemoglobina e são mais evidentes na presença de hipertensão arterial. Novas técnicas têm permitido avaliar a composição do miocárdio e a microcirculação coronariana. Alterações microvasculares podem levar a lesões de células miocárdicas com fibrose reacional e estão relacionadas a níveis elevados de glicose, à resistência à ação da insulina, disfunção endotelial e aumento da atividade simpática. Portanto, o uso da ecocardiografia com Doppler é útil para estabelecer o diagnóstico precoce de anormalidades cardíacas e avaliar se o efeito terapêutico de drogas cardioprotetoras (inibidores da enzima conversora da angiotensina, antagonistas do receptor da angiotensina II, antagonistas da aldosterona, beta-bloqueadores e estatinas) é capaz de impedir a progressão para estágios mais avançados de disfunção ventricular diastólica (9).

\section{Monitorização ambulatorial da pressão arterial}

A HAS é um dos principais fatores de risco para o desenvolvimento das complicações micro- e macrovasculares do diabetes. A HAS é prevalente nos pacientes com DM tipo 2, estando presente em 30\% no momento do diagnóstico do DM e em até $70 \%$ quando a ND já está estabelecida $(42,43)$. A realização da monitorização ambulatorial da pressão arterial (MAPA) tem sido recomendada para a avaliação do tratamento antihipertensivo em situações especiais, como em pacientes com HAS resistente ao tratamento, ou para detectar episódios hipotensivos induzidos por droga ou relacionados à neuropatia autonômica (44). Além disto, a MAPA permite a realização da análise de parâmetros até então impossíveis de serem registrados através da medida de PA no consultório, como as médias de PA sistólica e diastólica de 24 h, do dia e da noite; as cargas pressóricas e a ausência de descenso noturno da PA (45). Adicionalmente, permite a identificação de duas novas categorias de pacientes: com HAS do avental branco e com HAS mascarada. HAS mascarada inclui os indivíduos com PA normal no consultório $(\mathrm{PA}<140 / 90 \mathrm{~mm} \mathrm{Hg}$ ), mas classificados como hipertensos na medida da PA diurna da MAPA $(\geq 135 / 80$ $\mathrm{mm} \mathrm{Hg}$ ), que representam $10-20 \%$ dos indivíduos com PA normal no consultório (46). Nestes pacientes há também um aumento da EUA e da espessura do ventrículo esquerdo (5). Portanto, esses dados sugerem que a realização da MAPA é um procedimento importante em todos os pacientes diabéticos, especialmente naqueles com ND.

\section{PREVENÇÃO E TRATAMENTO}

A base para prevenção e tratamento da ND e, conseqüentemente, da DCV associada, é o tratamento agressivo dos fatores de risco que ambas apresentam em comum: hiperglicemia, hipertensão arterial e dislipidemia. As metas e estratégias a serem adotadas para promover proteção renal e $\mathrm{CV}$ em pacientes com ND estão apresentadas na tabela 2. Fatores adicionais desfavoráveis são a presença da anemia e o tabagismo.

\section{Controle glicêmico intensificado}

A obtenção de níveis glicêmicos próximos do ideal $\left(\mathrm{Al}_{\mathrm{c}}<7 \%\right)$ comprovadamente reduz o risco de desenvolver ND em cerca de $40 \%$ nos pacientes com DM tipo 1 (47) e $30 \%$ nos com tipo 2 (48).

Os efeitos do controle glicêmico intensificado na progressão da micro para a macroalbuminúria ainda não estão firmemente estabelecidos. No entanto, pacientes com insuficiência renal crônica em tratamento com hemodiálise e com níveis de $\mathrm{Al}_{\mathrm{c}}>8 \%$ apresentaram um aumento da mortalidade em um estudo de 7 anos de acompanhamento (49).

Os resultados do UKPDS sugerem que sulfoniluréias e metformina sejam comparáveis em termos de prevenção da ND, e que esta prevenção seria resultado do melhor controle metabólico obtido durante o tratamento. A metformina teria vantagem particular quanto à redução de mortalidade macrovascular em pacientes obesos. Contudo, nesses pacientes não houve redução de eventos microvasculares, incluindo a ND (48).

Alguns agentes anti-hiperglicêmicos orais poderiam ser úteis na prevenção da progressão de micro- para macroalbuminúria. A rosiglitazona, quando comparada à gliburida, demonstrou redução da EUA em pacientes com DM tipo 2, sugerindo um 
Tabela 2. Estratégias e metas para obtenção de proteção renal e cardiovascular em pacientes com nefropatia diabética.

\begin{tabular}{|c|c|c|}
\hline \multirow[t]{2}{*}{ Intervenção } & \multicolumn{2}{|c|}{ Metas } \\
\hline & Microalbuminúria & Macroalbuminúria \\
\hline IECA e/ou ARA II & $\begin{array}{l}\text { Redução da EUA ou reversão } \\
\text { para normoalbuminúria }\end{array}$ & $\begin{array}{c}\text { Proteinúria o mais baixo possível } \\
\qquad \text { ou }<0,5 \mathrm{~g} / 24 \mathrm{~h}\end{array}$ \\
\hline e dieta hipoprotéica & & \\
\hline$(0,6-0,8 \mathrm{~g} / \mathrm{kg} / \mathrm{dia}) *$ & Estabilização da TFG & Declínio da TFG $<2 \mathrm{ml} / \mathrm{min} /$ ano \\
\hline Controle pressórico & \multicolumn{2}{|c|}{$\mathrm{PA}<130 / 80$ ou $<125 / 75 \mathrm{~mm} \mathrm{Hg} * *$} \\
\hline Controle glicêmico & \multicolumn{2}{|c|}{$\mathrm{HbA} 1_{c}<7 \%$} \\
\hline Estatinas & \multicolumn{2}{|c|}{ LDL-C $\leq 100 \mathrm{mg} / \mathrm{dl} \#$} \\
\hline Ácido acetilsalicílico & \multicolumn{2}{|c|}{ Prevenção de trombose } \\
\hline Suspensão do fumo & \multicolumn{2}{|c|}{ Prevenção da progressão da aterosclerose } \\
\hline Anemia & \multicolumn{2}{|c|}{$\mathrm{Hb}>11<13 \mathrm{~g} / \mathrm{dl}$} \\
\hline
\end{tabular}

IECA= inibidores da enzima conversora; ARA II= antagonistas do receptor da angiotensina II; TFG= taxa de filtração glomerular; $\mathrm{PA}=$ pressão arterial; LDL-C= low density lipoprotein-cholesterol.

* Não comprovado benefício a longo prazo em pacientes microalbuminúricos.

* PA < 125/75 mmHg: na presença de creatinina sérica elevada e proteinúria > 1,0 g/24 h.

\# LDL-C $<70 \mathrm{mg} / \mathrm{dl}$ na presença de doença cardiovascular.

possível efeito benéfico na prevenção das complicações renais do DM tipo 2 (50). Em pacientes diabéticos tipo 2 em hemodiálise, a rosiglitazona melhorou o controle glicêmico, mas aumentou a retenção hídrica nos intervalos entre sessões (51). No estudo PROspective pioglitAzone Clinical Trial in macroVascular Events (PROACTIVE), o uso de pioglitazona em pacientes com creatinina sérica elevada associou-se a um aumento da mortalidade e de eventos CVs (52). Portanto, o uso de tiazolidinedionas em pacientes com insuficiência renal deve ser feito com cuidado, levando-se em consideração principalmente o potencial de retenção hídrica.

Nos pacientes diabéticos com comprometimento da função renal, deve ser lembrado que o uso de agentes anti-hiperglicêmicos orais deve levar em conta a função renal. A metformina não deve ser utilizada com valores de creatinina sérica $>1,5 \mathrm{mg} / \mathrm{dl}$ devido ao risco de acidose lática $(53,54)$.

Sulfoniluréias e seus metabólitos, com exceção da glimepirida, têm excreção renal e não devem ser utilizados em pacientes com perda significativa de função renal (55).

A repaglinida (56) e a nateglinida (57) apresentam uma curta duração de ação, são excretadas independentemente da função renal e parecem ser seguras para uso em pacientes com diminuição da função renal. No entanto, como em fases mais avançadas da ND a produção de insulina endógena é reduzida, em geral os pacientes com DM tipo 2 necessitam usar insulina para melhorar o controle glicêmico.

\section{Controle intensivo da pressão arterial e bloqueio do sistema renina-angiotensina}

A redução da pressão arterial está associada à diminuição do surgimento da ND e à redução importante do risco CV. No UKPDS, uma redução de 154 para $144 \mathrm{mmHg}$ na pressão arterial sistólica reduziu o risco de desenvolvimento da microalbuminúria em 29\% (58). No estudo Hypertension Optimal Treatment (HOT), uma redução na pressão diastólica de 85 para $81 \mathrm{mmHg}$ resultou em $50 \%$ de redução de risco para eventos CVs em pacientes com DM (59). Portanto, recomenda-se para os pacientes diabéticos em geral que os níveis de pressão arterial sejam inferiores a 130/80 $\mathrm{mmHg}(60)$. Quando os pacientes já apresentam micro- ou macroalbuminúria, os princípios de tratamento são os mesmos adotados para a prevenção primária, porém estratégias terapêuticas múltiplas visando remover os fatores de risco presentes e mais intensificadas devem ser empregadas nessas etapas.

Em pacientes com proteinúria, deve-se procurar atingir valores de pressão arterial mais baixos. De acordo com o estudo MDRD, quanto menor a pressão arterial, maior a preservação da função renal (61). Pacientes com proteinúria $>1 \mathrm{~g} /$ dia e insuficiência renal apresentavam menor declínio na função renal quando a pressão arterial era $<125 / 75 \mathrm{mmHg}$ (61). Apesar de este estudo ter incluído principalmente pacientes nãodiabéticos, esse alvo tem sido recomendado para pacientes com DM e proteinúria (62). 
O papel do bloqueio do sistema reninaangiotensina (SRA) na prevenção da ND ainda não está completamente definido, mas em pacientes com DM tipo 2, o uso de IECA e de antagonistas dos receptores da angiotensina II (ARA II) diminuíram o aparecimento de ND (63-65) e também o número de eventos CVs (66). No estudo Heart Outcomes Prevention Evaluation (MICRO-HOPE) (66), o ramipril $(10 \mathrm{mg} / \mathrm{dia})$ diminuiu o risco de nefropatia clínica em $24 \%$ e o risco de morte CV em 37\% nos pacientes com DM tipo 2 acima de 55 anos de idade com um fator de risco CV adicional. Portanto, os IECA têm demonstrado benefício na prevenção da doença renal e cardioproteção nos pacientes com DM tipo 2.

Nos pacientes com DM tipo 1 e tipo 2 microalbuminúricos, diversos estudos demonstraram que o tratamento da hipertensão, independentemente do agente utilizado, apresenta efeito benéfico sobre a albuminúria (67). O bloqueio do SRA com IECA ou ARA II confere um benefício adicional sobre a função renal. Este efeito renoprotetor é independente da redução da pressão arterial $(67,68)$ e está relacionado à redução da pressão intraglomerular e da passagem de proteínas no túbulo proximal (69). Essas drogas diminuem a EUA e a progressão da microalbuminúria para estágios mais avançados da ND.

Uma meta-análise de 12 ensaios clínicos avaliou o benefício do uso de IECA em indivíduos com DM tipo 1 e microalbuminúria, confirmando a redução de $60 \%$ de progressão para macroalbuminúria (70). Ensaios clínicos conduzidos em pacientes com DM tipo 2 microalbuminúricos também demonstraram o efeito nefroprotetor dos ARA II. Irbesartan $300 \mathrm{mg} /$ dia reduziu o risco de progressão para nefropatia clínica em 70\% em pacientes com DM tipo 2, hipertensos e microalbuminúricos, em um estudo de 2 anos de acompanhamento (71). Além disso, um achado interessante foi o de que, após 1 mês de suspensão dessa droga, os valores da EUA permaneciam reduzidos (72). Em outro ensaio, o Valsartan ( $80 \mathrm{mg} / \mathrm{dia})$ promoveu uma maior redução na EUA do que a amlodipina (44 vs. $8 \%$ ) com a mesma redução da pressão arterial (68). Esses dados reforçam a idéia de que o efeito anti-proteinúrico do ARA II é independente da pressão arterial. A comparação do efeito renoprotetor do IECA com o ARA II também já foi demonstrada. Um estudo com um ano de acompanhamento demonstrou redução similar da albuminúria com a utilização do losartan e do enalapril em pacientes com DM tipo 2 hipertensos e com microalbuminúria (73). Em um estudo prospectivo de 5 anos de acompanhamento, Barnett e col. (74) observaram que o telmisartan $(80 \mathrm{mg} /$ dia $)$ não foi inferior ao enalapril $(20 \mathrm{mg} /$ dia $)$ na prevenção do declínio da TFG em 250 pacientes com DM tipo 2 com microalbuminúria, sugerindo que os IECA e os ARA II têm um efeito similar na renoproteção. Recentemente, foi realizada uma meta-análise com 43 ensaios clínicos randomizados, que analisou o efeito do IECA e do ARA II isoladamente, assim como a comparação entre esses dois fármacos, sobre a progressão da ND e sobre a redução de mortalidade em pacientes com DM tipo 1 e tipo 2 com microe/ou macroalbuminúria (75). Esse estudo demonstrou que ambas as classes de fármacos apresentavam um efeito equivalente sobre a progressão da ND. Os IECA também apresentaram benefício na prevenção de mortalidade precoce. Entretanto, a comparação entre o IECA e o ARA II quanto ao efeito sobre mortalidade não foi possível, por falta de ensaios adequados para essa análise. Portanto, o uso de IECA ou ARA II é recomendado para todos os pacientes com DM tipo 1 e tipo 2 com microalbuminúria, mesmo que normotensos (76).

Nos pacientes proteinúricos, Mogensen (77) já havia demonstrado, há quase 30 anos, que o tratamento da HAS reduzia a albuminúria e a taxa de declínio da TFG em pacientes com DM tipo 1. Posteriormente, outros estudos claramente demonstraram que o tratamento agressivo da HAS apresenta um efeito benéfico na redução da queda da TFG em pacientes com DM tipo 1 e proteinúria (78). A adição dos IECA nos pacientes com DM tipo 1 e proteinúria (79) ou ARA II nos pacientes com DM tipo 2 macroalbuminúricos $(80,81)$ levou à redução da proteinúria e da perda de função renal. Apesar de não ter sido demonstrada diferença na taxa de eventos CVs, uma menor incidência de insuficiência cardíaca congestiva foi observada entre os pacientes com ARA II (80). Com a administração dos IECA para pacientes proteinúricos com creatinina sérica $>1,4 \mathrm{mg} / \mathrm{dl}$, pode ocorrer elevação da creatinina de até 30-35\%, estabilizando após 2 meses. Esse aumento é associado a uma preservação em longo prazo da função renal, e, portanto, nessa situação os IECA não devem ser suspensos (82). Elevações maiores de creatinina devem levantar a suspeita de estenose de artéria renal. Finalmente, a inibição do SRA, especialmente com os IECA, pode elevar os níveis de potássio sérico em pacientes com DM, principalmente na presença de insuficiência renal (83). Por esta razão, a EUA, a creatinina e o potássio séricos devem ser avaliados mensalmente nos primeiros 2 a 3 meses do início do uso de IECA ou ARA II. Recentemente, Mogensen e 
cols. (84) desenvolveram um novo conceito de duplo bloqueio do SRA. O IECA e ARA II interrompem o SRA em níveis diferentes, e a combinação destas classes de drogas pode ter um efeito sinergístico na redução da pressão arterial e da EUA em pacientes com DM tipo 1 e tipo 2 com ND (85). Apesar de não haver ainda estudos em longo prazo analisando o benefício do duplo bloqueio do SRA na ND, em pacientes proteinúricos não-diabéticos, o estudo Combination Treatment of Angiotensin-II Receptor Blocker and Angiotensin-Converting-Enzyme Inhibition in Nondiabetic Renal Disease (COOPERATE) demonstrou que o tratamento duplo foi superior à monoterapia nas doses máximas quanto a retardar a progressão da doença renal em um período de acompanhamento de 3 anos (86). A combinação de espironolactona, antagonista da aldosterona, com o IECA foi também mais efetiva na redução da EUA e da pressão arterial nos pacientes com DM tipo 2 micro- e macroalbuminúricos do que a utilização do IECA isoladamente (87).

\section{Estratégias no tratamento anti-hipertensivo nos pacientes com ND}

Revisões e recomendações recentes em relação aos agentes anti-hipertensivos utilizados no tratamento da HAS no paciente com ND estão disponíveis na literatura $(44,60,62,88,89)$. Para alcançar o alvo de pressão arterial recomendado de $130 / 80 \mathrm{mmHg}$ no paciente com DM em geral (60) e 125/75 mmHg nos pacientes com proteinúria $>1 \mathrm{~g}$ e aumento da creatinina sérica, são usualmente necessários 3 a 4 agentes anti-hipertensivos (90). Considera-se mais importante alcançar os níveis de pressão arterial recomendados do que particularmente o tipo de anti-hipertensivo utilizado, já que a maioria dos pacientes necessitará de vários fármacos. Entretanto, devido ao conhecido efeito nefroprotetor dos IECA e dos ARA II, o tratamento deve ser iniciado com um desses dois agentes. Os pacientes com pressão arterial sistólica $20 \mathrm{mmHg}$ e pressão arterial diastólica $10 \mathrm{mmHg}$ acima do alvo devem já iniciar com dois agentes anti-hipertensivos: um IECA ou um ARA II associado ao diurético hidroclorotiazida em baixa dose $(12,5$ a $25 \mathrm{mg} / \mathrm{dia})$ podem ser inicialmente utilizados.

Naqueles pacientes com a TFG $<30 \mathrm{ml} / \mathrm{min}$ (creatinina sérica de $2,5-3,0 \mathrm{mg} / \mathrm{dl}$ ), é indicada a utilização de diurético de alça (furosemide) (60). Na presença de efeitos colaterais dos IECA, como tosse, os ARA II são uma excelente alternativa e são os agentes preferidos para os pacientes com DM tipo 2 com hipertrofia ventricular esquerda (63) e/ou micro- ou macroalbuminúria $(66,71,80)$. No caso de não ocorrer redução da albuminúria ou de não se alcançar os níveis-alvos de pressão arterial, o IECA e o ARA II podem ser combinados, mesmo antes de se maximizar as doses de cada agente. Outros agentes anti-hipertensivos adicionais devem ser utilizados conforme a necessidade. Os bloqueadores do canal de cálcio têm um efeito adicional na redução dos níveis de pressão arterial. Esses agentes devem ser utilizados apenas em combinação com o IECA e não podem ser utilizados em pacientes com evento coronariano recente. Os beta-bloqueadores são especialmente indicados em pacientes com cardiopatia isquêmica comprovada, por estes fármacos reduzirem eventos CVs e mortalidade nos pacientes com freqüência cardíaca $>84$ bpm (90). Um composto neutro metabolicamente, carvedilol, deve ser utilizado. A combinação de beta-bloqueadores e bloqueadores do canal de cálcio do tipo não-dihidropiridinas não é recomendado, por ambos agentes apresentarem efeito cronotrópico negativo.

\section{Intervenção dietética}

A substituição da carne vermelha pela carne de galinha da dieta em curto prazo mostrou-se capaz de reduzir a EUA, assim como os níveis séricos de colesterol total, LDL e apolipoproteína B em pacientes com DM tipo 2 microalbuminúricos (91). Provavelmente esse efeito seja relacionado ao menor conteúdo de gordura saturada e maior proporção de ácidos graxos poliinsaturados observados na carne de galinha, quando comparada à carne vermelha. $\mathrm{O}$ efeito benéfico dos ácidos graxos poliinsaturados sobre a função endotelial (92) poderia também reduzir a EUA. Portanto, uma dieta normoprotéica com carne de galinha como única fonte de carne pode representar uma estratégia aditiva para o tratamento dos pacientes com DM tipo 2 e microalbuminúria. No entanto, são necessários estudos em longo prazo para confirmar esse efeito favorável. De acordo com uma meta-análise realizada com 5 estudos, incluindo um total de 108 pacientes com DM tipo 1, a restrição protéica da dieta retardou a progressão da ND (93). Mais recentemente, um ensaio clínico randomizado e controlado, com 84 pacientes com DM tipo 1 com ND acompanhados por 4 anos, demonstrou que a adoção de dieta com moderada restrição protéica $(0,9 \mathrm{~g} / \mathrm{kg} / \mathrm{dia})$ reduziu o risco de insuficiência renal crônica terminal ou morte em $76 \%$, apesar de não ter tido efeito sobre o declínio da TFG (94). O mecanismo através do qual a dieta hipoprotéica pode reduzir a progressão da ND é desconhecido, mas pode estar relacionado à melhora do perfil lipídico e/ou da hemodinâmica glomerular. 


\section{Dislipidemia}

O nível-alvo desejado do colesterol LDL é $<100$ $\mathrm{mg} / \mathrm{dl}$ para pacientes com DM em geral e $<70 \mathrm{mg} / \mathrm{dl}$ na presença de doença CV (95). O efeito da diminuição dos lipídios séricos com drogas hipolipemiantes sobre a progressão da ND é ainda desconhecido. Uma meta-análise recente, envolvendo 27 estudos e 39.704 participantes, sugere que ocorra uma redução modesta da proteinúria com o uso de estatinas. Entretanto, em relação à proteção no declínio da TFG, somente os subgrupos com doença $\mathrm{CV}$ se beneficiaram, o que não foi o caso dos indivíduos com DM (96). Outra metaanálise também recente, envolvendo 15 ensaios clínicos randomizados, controlados com placebo, sendo 8 em indivíduos com DM, confirma que as estatinas de fato reduzem a albuminúria, de forma mais acentuada nos níveis urinários mais elevados. No entanto, conclui-se pela necessidade de ensaios maiores para definir o benefício CV e renal final dessas drogas na nefropatia (97). De qualquer maneira, e de forma independente do status renal, os resultados do Collaborative Atorvastatin Diabetes Study (CARDS) demonstram uma marcada redução nos eventos CVs em pacientes com DM e pelo menos um fator de risco adicional para doença coronariana, sugerindo que todos os pacientes com DM deveriam utilizar estatinas (98).

\section{Anemia}

A anemia pode estar presente nos pacientes com ND mesmo antes de apresentarem perda de função renal avançada (creatinina sérica $<1,8 \mathrm{mg} / \mathrm{dl}$ ), e parece estar relacionada à deficiência de eritropoietina (99). Além disso, a anemia tem sido considerada um fator de risco para a progressão da doença renal, assim como da retinopatia (100). Enquanto não se tem os resultados do ensaio clínico em andamento Anemia Correction in Diabetes (ACORD) (101) e do TREAT, recomenda-se iniciar a reposição de eritropoietina quando os níveis de hemoglobina estiverem < $11 \mathrm{~g} / \mathrm{dl}$. Os níveis desejáveis de hemoglobina devem ser 12-13 g/dl. Durante o tratamento com eritropoietina, deve-se levar em consideração o risco potencial de elevação dos níveis pressóricos (100).

No entanto, deve-se ter em conta, a partir da experiência em pacientes não-diabéticos, que a correção da anemia com eritropoietina pode não oferecer os resultados esperados. Particularmente quando a hemoglobina se eleva acima de $13 \mathrm{~g} / \mathrm{dl}$ pode haver aumento da mortalidade, inclusive CV (102). Até que mais estudos estejam disponíveis, deve-se exercer cautela para não tentar corrigir completamente a anemia em pacientes renais crônicos.

\section{Uso de aspirina}

Aspirina em baixa dosagem tem sido recomendada para a prevenção primária e secundária de eventos CVs em adultos com DM. O temido impacto negativo sobre a função renal (EUA ou TFG) não tem sido observado em pacientes com DM tipo 1 e tipo $2 \mathrm{com}$ micro- e macroalbuminúria (101,103). Além disso, não tem sido encontrada interação farmacológica desfavorável com o uso simultâneo de IECA em pacientes microalbuminúricos, quando a aspirina supostamente poderia diminuir os efeitos antiproteinúricos dos IECA (104). Outro aspecto é o de que uma análise do ensaio Primary Prevention Project não mostrou uma redução significativa na ocorrência de eventos CVs em um subgrupo de 1.031 pacientes com DM utilizando aspirina em baixa dosagem (100 $\mathrm{mg} /$ dia) (105). Apesar de este estudo não apresentar poder estatístico para analisar o efeito sobre o desenvolvimento de eventos CVs, esses dados sugerem que os pacientes com DM possam ser menos responsivos ao tratamento com aspirina ("resistência à aspirina"). De fato, um estudo recente demonstrou uma resposta reduzida das plaquetas de indivíduos com DM ao tratamento com aspirina ( $150 \mathrm{mg} / \mathrm{dia})$. Esse fenômeno foi associado a maiores valores de glico-hemoglobina, menores níveis séricos de colesterol HDL e maiores níveis de colesterol total (106). Portanto, os pacientes com DM possivelmente se beneficiem de doses de aspirina > 100-150 mg/dia ou de outros agentes anti-plaquetários como o clopidogrel.

\section{Intervenção multifatorial}

Os pacientes com microalbuminúria freqüentemente apresentam outros fatores de risco CVs, como HAS e dislipidemia. No estudo Steno-2, uma intervenção multifatorial foi comparada ao tratamento convencional em 160 pacientes com DM tipo 2 microalbuminúricos (107). As metas da intervenção multifatorial eram alcançar valores de pressão arterial abaixo de $130 / 80 \mathrm{mmHg}$, níveis de colesterol total sérico < $175 \mathrm{mg} / \mathrm{dl}$, valores de triglicerídeos séricos < 150 $\mathrm{mg} / \mathrm{dl}$ e valores de glico-hemoglobina $<6,5 \%$. A intervenção multifatorial consistiu da implementação de modificações de estilo de vida e tratamento farmacológico, incluindo uma dieta pobre em gordura, programa de exercícios físicos leves a moderados 3 a 5 vezes por semana, programa para cessar de fumar e prescrição de IECA ou ARA II e aspirina. O grupo de pacientes com tratamento intensificado apresentou uma redução do risco de desenvolver macroalbuminúria de $61 \%$ e uma redução no risco de retinopatia e neuropatia 
autonômica de 58 e $63 \%$, respectivamente. Além disso, o achado mais importante foi uma redução de $55 \%$ no risco de desenvolvimento de desfechos compostos que consistiam de morte por doença $\mathrm{CV}$, infarto do miocárdio não-fatal, procedimentos de revascularização, acidente vascular cerebral não-fatal e amputação, no grupo de pacientes com a intervenção mutifatorial.

\section{CONSIDERAÇÕES FINAIS}

Pacientes em diferentes estágios de ND apresentam freqüentemente comprometimento cardíaco expresso por isquemia miocárdica e/ou cardiomiopatia diabética. Estas alterações já estão presentes em estágios iniciais da ND e provavelmente mesmo antes de a EUA atingir níveis tradicionalmente diagnósticos de microalbuminúria. As alterações cardíacas são responsáveis por uma proporção significativa de mortes nos pacientes com ND e podem ser reduzidas através de intervenção nos múltiplos fatores de risco cardiovascular encontrados nesses pacientes. A avaliação de doença cardíaca deve idealmente ser realizada em todos os pacientes com qualquer grau de ND através de métodos específicos para detectar isquemia e disfunção miocárdica, além do emprego rotineiro da MAPA. Em pacientes com aterosclerose avançada também devem ser avaliadas outras artérias (carótidas, aorta, renais). $\mathrm{O}$ tratamento rigoroso da hipertensão arterial, o uso de fármacos cardioprotetores, o tratamento da dislipidemia e da anemia, assim como o emprego de medicamentos anti-plaquetários, poderão reduzir a elevada mortalidade cardiovascular na ND.

\section{REFERÊNCIAS}

1. Deckert T, Poulsen JE, Larsen M. Prognosis of diabetics with diabetes onset before the age of thirty-one. I. Survival, causes of death, and complications. Diabetologia 1978;14:363-70.

2. Gross JL, de Azevedo MJ, Silveiro SP, Canani LH, Caramori $\mathrm{ML}$, Zelmanovitz T. Diabetic nephropathy: diagnosis, prevention, and treatment. Diabetes Care 2005;28:164-76.

3. Adler Al, Stevens RJ, Manley SE, Bilous RW, Cull CA, Holman RR. Development and progression of nephropathy in type 2 diabetes: the United Kingdom Prospective Diabetes Study (UKPDS 64). Kidney Int 2003;63:225-32.

4. Beck MO, Silveiro SP, Friedman R, Clausell N, Gross JL. Asymptomatic coronary artery disease is associated with cardiac autonomic neuropathy and diabetic nephropathy in type 2 diabetic patients. Diabetes Care 1999;22:1745-7.

5. Leitao CB, Canani LH, Polson PB, Molon MP, Pinotti AF, Gross JL. Urinary albumin excretion rate is associated with increased ambulatory blood pressure in normoalbuminuric type 2 diabetic patients. Diabetes Care 2005;28:1724-9.

6. Murussi M, Baglio P, Gross JL, Silveiro SP. Risk factors for microalbuminuria and macroalbuminuria in type 2 diabetic patients: a 9-year follow-up study. Diabetes Care 2002; 25:1101-3.
7. Rachmani R, Levi Z, Lidar M, Slavachevski I, Half-Onn E, Ravid M. Considerations about the threshold value of microalbuminuria in patients with diabetes mellitus: lessons from an 8-year follow-up study of 599 patients. Diabetes Res Clin Pract 2000;49:187-94.

8. Kannel WB, Hjortland M, Castelli WP. Role of diabetes in congestive heart failure: the Framingham study. Am J Cardiol 1974;34:29-34.

9. Galderisi M. Diastolic dysfunction and diabetic cardiomyopathy: evaluation by Doppler echocardiography. J Am Coll Cardiol 2006;48:1548-51.

10. Yusuf S, Sleight P, Pogue J, Bosch J, Davies R, Dagenais G. Effects of an angiotensin-converting-enzyme inhibitor, ramipril, on cardiovascular events in high-risk patients. The Heart Outcomes Prevention Evaluation Study Investigators. N Engl J Med 2000;342:145-53.

11. Gerstein HC, Mann JF, Yi Q, Zinman B, Dinneen SF, Hoogwerf $B$, et al. Albuminuria and risk of cardiovascular events, death, and heart failure in diabetic and nondiabetic individuals. JAMA 2001;286:421-6.

12. Deckert T, Feldt-Rasmussen B, Borch-Johnsen $K$, Jensen $T$, Kofoed-Enevoldsen A. Albuminuria reflects widespread vascular damage. The Steno hypothesis. Diabetologia 1989; 32:219-26.

13. Dinneen SF, Gerstein HC. The association of microalbuminuria and mortality in non-insulin-dependent diabetes mellitus. A systematic overview of the literature. Arch Intern Med 1997; 157:1413-8.

14. Klausen K, Borch-Johnsen K, Feldt-Rasmussen B, Jensen G, Clausen $P$, Scharling $H$, et al. Very low levels of microalbuminuria are associated with increased risk of coronary heart disease and death independently of renal function, hypertension, and diabetes. Circulation 2004; 110:32-5.

15. Arnlov J, Evans JC, Meigs JB, Wang TJ, Fox CS, Levy D, et al. Low-grade albuminuria and incidence of cardiovascular disease events in nonhypertensive and nondiabetic individuals: the Framingham Heart Study. Circulation 2005;112:969-75.

16. Hansen KW, Mau Pedersen M, Marshall SM, Christiansen JS, Mogensen CE. Circadian variation of blood pressure in patients with diabetic nephropathy. Diabetologia 1992; 35:1074-9.

17. Jenkins AJ, Lyons TJ, Zheng D, Otvos JD, Lackland DT, McGee D, et al. Lipoproteins in the DCCT/EDIC cohort: associations with diabetic nephropathy. Kidney Int 2003;64:81728.

18. Perassolo MS, Almeida JC, Pra RL, Mello VD, Maia AL, Moulin CC, et al. Fatty acid composition of serum lipid fractions in type 2 diabetic patients with microalbuminuria. Diabetes Care 2003;26:613-8.

19. Costa LA, Canani LH, Lisboa HR, Tres GS, Gross JL. Aggregation of features of the metabolic syndrome is associated with increased prevalence of chronic complications in type 2 diabetes. Diabet Med 2004;21:252-5.

20. Stehouwer CD, Gall MA, Twisk JW, Knudsen E, Emeis JJ, Parving $\mathrm{HH}$. Increased urinary albumin excretion, endothelial dysfunction, and chronic low-grade inflammation in type 2 diabetes: progressive, interrelated, and independently associated with risk of death. Diabetes 2002;51:1157-65.

21. Murussi M, Gross JL, Silveiro SP. Glomerular filtration rate changes in normoalbuminuric and microalbuminuric type 2 diabetic patients and normal individuals. A 10-year follow-up. J Diabetes Complications 2006;20:210-5.

22. Ibsen $\mathrm{H}$, Olsen MH, Wachtell K, Borch-Johnsen K, Lindholm $\mathrm{LH}$, Mogensen CE, et al. Reduction in albuminuria translates to reduction in cardiovascular events in hypertensive patients: losartan intervention for endpoint reduction in hypertension study. Hypertension 2005;45:198-202.

23. Diercks GF, van Boven AJ, Hillege HL, Janssen WM, Kors JA, de Jong PE, et al. Microalbuminuria is independently associated with ischaemic electrocardiographic abnormalities in a large non-diabetic population. The PREVEND (Prevention of REnal and Vascular ENdstage Disease) study. Eur Heart $\mathbf{J}$ 2000;21:1922-7. 
24. Jamerson KA, Bakris GL, Wun CC, Dahlof B, Lefkowitz M, Manfreda S, et al. Rationale and design of the avoiding cardiovascular events through combination therapy in patients living with systolic hypertension (ACCOMPLISH) trial: the first randomized controlled trial to compare the clinical outcome effects of first-line combination therapies in hypertension. Am J Hypertens 2004; 17:793-801.

25. Knobler H, Zornitzki T, Vered S, Oettinger M, Levy R, Caspi A, et al. Reduced glomerular filtration rate in asymptomatic diabetic patients: predictor of increased risk for cardiac events independent of albuminuria. J Am Coll Cardiol 2004; 44:2142-8.

26. Viberti GC, Bilous RW, Mackintosh D, Keen H. Monitoring glomerular function in diabetic nephropathy. A prospective study. Am J Med 1983;74:256-64.

27. Gall MA, Nielsen FS, Smidt UM, Parving HH. The course of kidney function in type 2 (non-insulin-dependent) diabetic patients with diabetic nephropathy. Diabetologia 1993;36:1071-8.

28. Friedman R, Gross JL. Evolution of glomerular filtration rate in proteinuric NIDDM patients. Diabetes Care 1991;14:355-9.

29. Kim WY, Astrup AS, Stuber M, Tarnow L, Falk E, Botnar RM, et al. Subclinical coronary and aortic atherosclerosis detected by magnetic resonance imaging in type 1 diabetes with and without diabetic nephropathy. Circulation 2007; in press.

30. Astrup AS, Tarnow L, Rossing P, Hansen BV, Hilsted J, Parving $\mathrm{HH}$. Cardiac autonomic neuropathy predicts cardiovascular morbidity and mortality in type 1 diabetic patients with diabetic nephropathy. Diabetes Care 2006;29:334-9.

31. Bruno RM, Gross JL. Prognostic factors in Brazilian diabetic patients starting dialysis: a 3.6-year follow-up study. J Diabetes Complications 2000;14:266-71.

32. Zaslavsky LM, Pinotti AF, Gross JL. Diastolic dysfunction and mortality in diabetic patients on hemodialysis: a 4.25-year controlled prospective study. J Diabetes Complications 2005;19:194-200.

33. Budoff MJ, Achenbach S, Blumenthal RS, Carr JJ, Goldin JG, Greenland P, et al. Assessment of coronary artery disease by cardiac computed tomography: a scientific statement from the American Heart Association Committee on Cardiovascular Imaging and Intervention, Council on Cardiovascular Radiology and Intervention, and Committee on Cardiac Imaging, Council on Clinical Cardiology. Circulation 2006;114:1761-91.

34. Valabhji J, Robinson S, Poulter C, Robinson AC, Kong C, Henzen $\mathrm{C}$, et al. Prevalence of renal artery stenosis in subjects with type 2 diabetes and coexistent hypertension. Diabetes Care 2000;23:539-43.

35. Nicholls AJ. The impact of atherosclerotic renovascular disease on diabetic renal failure. Diabet Med 2002;19:889-94.

36. White CJ, Jaff MR, Haskal ZJ, Jones DJ, Olin JW, RochaSingh KJ, et al. Indications for renal arteriography at the time of coronary arteriography: a science advisory from the American Heart Association Committee on Diagnostic and Interventional Cardiac Catheterization, Council on Clinical Cardiology, and the Councils on Cardiovascular Radiology and Intervention and on Kidney in Cardiovascular Disease. Circulation 2006;114:1892-5.

37. Safian RD, Textor SC. Renal-artery stenosis. N Engl J Med 2001;344:431-42.

38. Barrett BJ, Parfrey PS. Clinical practice. Preventing nephropathy induced by contrast medium. N Engl J Med 2006;354:379-86.

39. Gross JL, Friedman R, Silveiro SP. Preventing nephropathy induced by contrast medium. N Engl J Med 2006;354:18535; author reply 1853-5.

40. Zagler A, Azadpour M, Mercado C, Hennekens CH. N-acetylcysteine and contrast-induced nephropathy: a meta-analysis of 13 randomized trials. Am Heart J 2006;151:140-5.

41. Poirier P, Bogaty P, Garneau C, Marois L, Dumesnil JG. Diastolic dysfunction in normotensive men with well-controlled type 2 diabetes: importance of maneuvers in echocardiographic screening for preclinical diabetic cardiomyopathy. Diabetes Care 2001;24:5-10.
42. Remuzzi G, Schieppati A, Ruggenenti P. Clinical practice. Nephropathy in patients with type 2 diabetes. N Engl J Med 2002;346:1145-51.

43. Scheffel RS, Bortolanza D, Weber CS, Costa LA, Canani LH, Santos KG, et al. Prevalence of micro and macroangiopathic chronic complications and their risk factors in the care of out patients with type 2 diabetes mellitus. Rev Assoc Med Bras 2004;50:263-7.

44. Working Party of the International Diabetes Federation (European Region). Hypertension in people with type 2 diabetes: knowledge-based diabetes-specific guidelines. Diabet Med 2003;20:972-87.

45. Pickering TG, Shimbo D, Haas D. Ambulatory blood-pressure monitoring. N Engl J Med 2006;354:2368-74.

46. III Guidelines for the Use of Ambulatory Blood Pressure Monitoring - ambulatory monitoring of blood pressure. Sociedade Brasileira de Hipertensão. Arq Bras Cardiol 2001;77:384-9.

47. The effect of intensive treatment of diabetes on the development and progression of long-term complications in insulindependent diabetes mellitus. The Diabetes Control and Complications Trial Research Group. N Engl J Med 1993;329:977-86.

48. Intensive blood-glucose control with sulphonylureas or insulin compared with conventional treatment and risk of complications in patients with type 2 diabetes (UKPDS 33). UK Prospective Diabetes Study (UKPDS) Group. Lancet 1998;352:837-53.

49. Oomichi T, Emoto M, Tabata T, Morioka T, Tsujimoto $Y$, Tahara $\mathrm{H}$, et al. Impact of glycemic control on survival of diabetic patients on chronic regular hemodialysis: a 7-year observational study. Diabetes Care 2006;29:1496-500.

50. Bakris G, Viberti G, Weston WM, Heise M, Porter LE, Freed MI. Rosiglitazone reduces urinary albumin excretion in type II diabetes. J Hum Hypertens 2003;17:7-12.

51. Chiang CK, Ho TI, Peng YS, Hsu SP, Pai MF, Yang SY, et al. Rosiglitazone in diabetes control in hemodialysis patients with and without viral hepatitis infection: effectiveness and side effects. Diabetes Care 2007;30:3-7.

52. Dormandy JA, Charbonnel B, Eckland DJ, Erdmann E, MassiBenedetti M, Moules IK, et al. Secondary prevention of macrovascular events in patients with type 2 diabetes in the PROactive Study (PROspective pioglitAzone Clinical Trial In macroVascular Events): a randomised controlled trial. Lancet 2005;366:1279-89.

53. Bailey CJ, Turner RC. Metformin. N Engl J Med 1996;334:574-9.

54. Holstein A, Stumvoll M. Contraindications can damage your health - is metformin a case in point? Diabetologia 2005;48:2454-9.

55. Inzucchi SE. Oral antihyperglycemic therapy for type 2 diabetes: scientific review. JAMA 2002;287:360-72.

56. Hasslacher C. Safety and efficacy of repaglinide in type 2 diabetic patients with and without impaired renal function. Diabetes Care 2003;26:886-91.

57. Del Prato S, Heine RJ, Keilson L, Guitard C, Shen SG, Emmons RP. Treatment of patients over 64 years of age with type 2 diabetes: experience from nateglinide pooled database retrospective analysis. Diabetes Care 2003;26:2075-80.

58. Tight blood pressure control and risk of macrovascular and microvascular complications in type 2 diabetes: UKPDS 38. UK Prospective Diabetes Study Group. BMJ 1998;317:70313.

59. Hansson L, Zanchetti A, Carruthers SG, Dahlof B, Elmfeldt D, Julius $S$, et al. Effects of intensive blood-pressure lowering and low-dose aspirin in patients with hypertension: principal results of the Hypertension Optimal Treatment (HOT) randomised trial. HOT Study Group. Lancet 1998;351:1755-62.

60. Chobanian AV, Bakris GL, Black HR, Cushman WC, Green LA, Izzo JL, Jr., et al. The seventh report of the Joint National Committee on prevention, detection, evaluation, and treatment of high blood pressure: the JNC 7 report. JAMA 2003;289:2560-72. 
61. Peterson JC, Adler S, Burkart JM, Greene T, Hebert LA, Hunsicker LG, et al. Blood pressure control, proteinuria, and the progression of renal disease. The Modification of Diet in Renal Disease Study. Ann Intern Med 1995;123:754-62.

62. Bakris GL, Williams M, Dworkin L, Elliott WJ, Epstein M, Toto $\mathrm{R}$, et al. Preserving renal function in adults with hypertension and diabetes: a consensus approach. National Kidney Foundation Hypertension and Diabetes Executive Committees Working Group. Am J Kidney Dis 2000;36:646-61.

63. Lindholm LH, Ibsen H, Dahlof B, Devereux RB, Beevers G, de Faire $U$, et al. Cardiovascular morbidity and mortality in patients with diabetes in the Losartan Intervention For Endpoint reduction in hypertension study (LIFE): a randomised trial against atenolol. Lancet 2002;359:1004-10.

64. Ravid M, Brosh D, Levi Z, Bar-Dayan Y, Ravid D, Rachmani R. Use of enalapril to attenuate decline in renal function in normotensive, normoalbuminuric patients with type 2 diabetes mellitus. A randomized, controlled trial. Ann Intern Med 1998; 128:982-8.

64. Ruggenenti $P$, Fassi $A$, llieva AP, Bruno $S$, lliev IP, Brusegan $\mathrm{V}$, et al. Preventing microalbuminuria in type 2 diabetes. $\mathbf{N}$ Engl J Med 2004;351:1941-51.

66. Effects of ramipril on cardiovascular and microvascular outcomes in people with diabetes mellitus: results of the HOPE study and MICRO-HOPE substudy. Heart Outcomes Prevention Evaluation Study Investigators. Lancet 2000;355:253-9.

67. Mogensen CE. Microalbuminuria and hypertension with focus on type 1 and type 2 diabetes. J Intern Med 2003;254:45-66.

68. Viberti G, Wheeldon NM. Microalbuminuria reduction with valsartan in patients with type 2 diabetes mellitus: a blood pressure-independent effect. Circulation 2002;106:672-8.

69. Thurman JM, Schrier RW. Comparative effects of angiotensin-converting enzyme inhibitors and angiotensin receptor blockers on blood pressure and the kidney. Am $\mathbf{J}$ Med 2003;114:588-98.

70. Should all patients with type 1 diabetes mellitus and microalbuminuria receive angiotensin-converting enzyme inhibitors? A meta-analysis of individual patient data. Ann Intern Med 2001;134:370-9.

71. Parving $H H$, Lehnert $H$, Brochner-Mortensen J, Gomis R, Andersen S, Arner P. The effect of irbesartan on the development of diabetic nephropathy in patients with type 2 diabetes. N Engl J Med 2001;345:870-8.

72. Andersen S, Brochner-Mortensen J, Parving HH. Kidney function during and after withdrawal of long-term irbesartan treatment in patients with type 2 diabetes and microalbuminuria. Diabetes Care 2003;26:3296-302.

73. Lacourciere Y, Belanger A, Godin C, Halle JP, Ross S, Wright $\mathrm{N}$, et al. Long-term comparison of losartan and enalapril on kidney function in hypertensive type 2 diabetics with early nephropathy. Kidney Int 2000;58:762-9.

74. Barnett AH, Bain SC, Bouter P, Karlberg B, Madsbad S, Jervell $J$, et al. Angiotensin-receptor blockade versus convertingenzyme inhibition in type 2 diabetes and nephropathy. $\mathbf{N}$ Engl J Med 2004;351:1952-61.

75. Strippoli GF, Craig M, Deeks JJ, Schena FP, Craig JC. Effects of angiotensin converting enzyme inhibitors and angiotensin II receptor antagonists on mortality and renal outcomes in diabetic nephropathy: systematic review. BMJ 2004; 329:828.

76. Molitch ME, DeFronzo RA, Franz MJ, Keane WF, Mogensen $\mathrm{CE}$, Parving $\mathrm{HH}$, et al. Nephropathy in diabetes. Diabetes Care 2004;27(suppl 1):S79-83.

77. Mogensen CE. Progression of nephropathy in long-term diabetics with proteinuria and effect of initial anti-hypertensive treatment. Scand J Clin Lab Invest 1976;36:383-8.

78. Parving HH, Andersen AR, Smidt UM, Svendsen PA. Early aggressive antihypertensive treatment reduces rate of decline in kidney function in diabetic nephropathy. Lancet 1983;1:1175-9.

79. Lewis EJ, Hunsicker LG, Bain RP, Rohde RD. The effect of angiotensin-converting-enzyme inhibition on diabetic nephropathy. The Collaborative Study Group. N Engl J Med 1993;329:1456-62.
80. Brenner BM, Cooper ME, de Zeeuw D, Keane WF, Mitch WE, Parving $\mathrm{HH}$, et al. Effects of losartan on renal and cardiovascular outcomes in patients with type 2 diabetes and nephropathy. N Engl J Med 2001;345:861-9.

81. Lewis EJ, Hunsicker LG, Clarke WR, Berl T, PohI MA, Lewis $\mathrm{JB}$, et al. Renoprotective effect of the angiotensin-receptor antagonist irbesartan in patients with nephropathy due to type 2 diabetes. N Engl J Med 2001;345:851-60.

82. Bakris GL, Weir MR. Angiotensin-converting enzyme inhibitor-associated elevations in serum creatinine: is this a cause for concern? Arch Intern Med 2000;160:685-93.

83. Bakris GL, Siomos M, Richardson D, Janssen I, Bolton WK, Hebert $L$, et al. ACE inhibition or angiotensin receptor blockade: impact on potassium in renal failure. VAL-K Study Group. Kidney Int 2000;58:2084-92.

84. Andersen NH, Poulsen PL, Knudsen ST, Poulsen SH, Eiskjaer $\mathrm{H}$, Hansen $\mathrm{KW}$, et al. Long-term dual blockade with candesartan and lisinopril in hypertensive patients with diabetes: the CALM II study. Diabetes Care 2005;28:273-7.

85. Jacobsen $\mathrm{P}$, Rossing K, Parving HH. Single versus dual blockade of the renin-angiotensin system (angiotensin-converting enzyme inhibitors and/or angiotensin II receptor blockers) in diabetic nephropathy. Curr Opin Nephrol Hypertens 2004; 13:319-24.

86. Nakao N, Yoshimura A, Morita H, Takada M, Kayano T, Ideura T. Combination treatment of angiotensin-II receptor blocker and angiotensin-converting-enzyme inhibitor in non-diabetic renal disease (COOPERATE): a randomised controlled trial. Lancet 2003;361:117-24.

87. Sato A, Hayashi K, Naruse M, Saruta T. Effectiveness of aldosterone blockade in patients with diabetic nephropathy. Hypertension 2003;41:64-8.

88. Vijan S, Hayward RA. Treatment of hypertension in type 2 diabetes mellitus: blood pressure goals, choice of agents, and setting priorities in diabetes care. Ann Intern Med 2003;138:593-602.

89. Jandeleit-Dahm K, Cooper ME. Hypertension and diabetes. Curr Opin Nephrol Hypertens 2002;11:221-8.

90. Bakris GL. A practical approach to achieving recommended blood pressure goals in diabetic patients. Arch Intern Med 2001;161:2661-7.

91. Gross JL, Zelmanovitz T, Moulin CC, De Mello V, Perassolo $M$, Leitão $C$, et al. Effect of a chicken-based diet on renal function and lipid profile in patients with type 2 diabetes: a randomized crossover trial. Diabetes Care 2002;25:645-51.

92. Ros E, Nunez I, Perez-Heras A, Serra M, Gilabert R, Casals E, et al. A walnut diet improves endothelial function in hypercholesterolemic subjects: a randomized crossover trial. Circulation 2004; 109:1609-14.

93. Pedrini MT, Levey AS, Lau J, Chalmers TC, Wang PH. The effect of dietary protein restriction on the progression of diabetic and nondiabetic renal diseases: a meta-analysis. Ann Intern Med 1996;124:627-32.

94. Hansen HP, Tauber-Lassen E, Jensen BR, Parving HH. Effect of dietary protein restriction on prognosis in patients with diabetic nephropathy. Kidney Int 2002;62:220-8.

95. Grundy SM, Cleeman JI, Merz CN, Brewer HB, Jr., Clark LT, Hunninghake DB, et al. Implications of recent clinical trials for the National Cholesterol Education Program Adult Treatment Panel III guidelines. Circulation 2004;110:227-39.

96. Sandhu S, Wiebe N, Fried LF, Tonelli M. Statins for improving renal outcomes: a meta-analysis. J Am Soc Nephrol 2006;17:2006-16.

97. Douglas K, O'Malley PG, Jackson JL. Meta-analysis: the effect of statins on albuminuria. Ann Intern Med 2006;145:11724.

98. Colhoun HM, Betteridge DJ, Durrington PN, Hitman GA, Neil $\mathrm{HA}$, Livingstone SJ, et al. Primary prevention of cardiovascular disease with atorvastatin in type 2 diabetes in the Collaborative Atorvastatin Diabetes Study (CARDS): multicentre randomised placebo-controlled trial. Lancet 2004;364:685-96.

99. Sinclair SH, DelVecchio C, Levin A. Treatment of anemia in the diabetic patient with retinopathy and kidney disease. Am J Ophthalmol 2003;135:740-3. 
100.Laville M. New strategies in anaemia management: ACORD (Anaemia CORrection in Diabetes) trial. Acta Diabetol 2004;41(suppl 1):S18-22

101.Gaede $\mathrm{P}$, Hansen HP, Parving HH, Pedersen O. Impact of lowdose acetylsalicylic acid on kidney function in type 2 diabetic patients with elevated urinary albumin excretion rate. Nephrol Dial Transplant 2003;18:539-42.

102.Remuzzi G, Ingelfinger JR. Correction of anemia - payoffs and problems. N Engl J Med 2006;355:2144-6.

103. Hansen HP, Gaede PH, Jensen BR, Parving HH. Lack of impact of low-dose acetylsalicylic acid on kidney function in type 1 diabetic patients with microalbuminuria. Diabetes Care 2000;23:1742-5.

104.Camargo EG, Weinert LS, Lavinski J, Gross JL, Silveiro SP. Low-dose aspirin does not affect antiproteinuric effect of enalapril in microalbuminuric type 2 diabetic patients. A randomized, double -blind, cross-over study. Diabetologia 2006;49(S):639.

105.Sacco M, Pellegrini F, Roncaglioni MC, Avanzini F, Tognoni G, Nicolucci A. Primary prevention of cardiovascular events with low-dose aspirin and vitamin E in type 2 diabetic patients: results of the Primary Prevention Project (PPP) trial. Diabetes Care 2003;26:3264-72.
106.Watala C, Golanski J, Pluta J, Boncler M, Rozalski M, Luzak B, et al. Reduced sensitivity of platelets from type 2 diabetic patients to acetylsalicylic acid (aspirin)-its relation to metabolic control. Thromb Res 2004:113:101-13.

107.Gaede P, Vedel P, Larsen N, Jensen GV, Parving HH, Pedersen $\mathrm{O}$. Multifactorial intervention and cardiovascular disease in patients with type 2 diabetes. N Engl J Med 2003; 348:383-93.

Endereço para correspondência:

Jorge Luiz Gross

Serviço de Endocrinologia

Hospital de Clínicas de Porto Alegre

Rua Ramiro Barcelos 2350, 4० andar, Prédio 12

90035-903 Porto Alegre, RS 